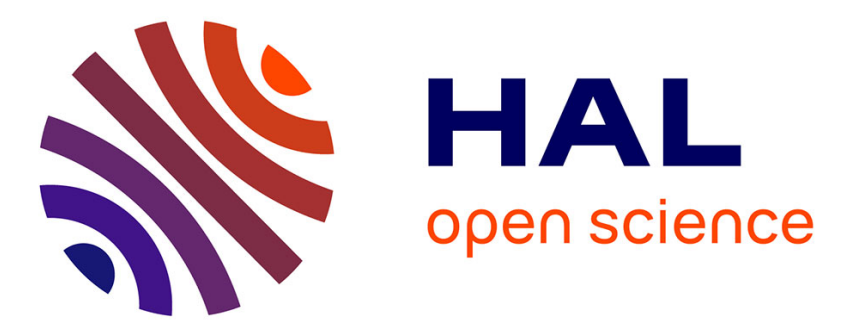

\title{
Simulating Patient Specific Multiple Time-point MRIs From a Biophysical Model of Brain Deformation in Alzheimer's Disease
}

\author{
Bishesh Khanal, Marco Lorenzi, Nicholas Ayache, Xavier Pennec
}

\section{- To cite this version:}

Bishesh Khanal, Marco Lorenzi, Nicholas Ayache, Xavier Pennec. Simulating Patient Specific Multiple Time-point MRIs From a Biophysical Model of Brain Deformation in Alzheimer's Disease. Workshop on Computational Biomechanics for Medicine - X, Oct 2015, Munich, France. pp.167-176, 10.1007/978-3-319-28329-6_15 . hal-01217080

\author{
HAL Id: hal-01217080 \\ https://hal.inria.fr/hal-01217080
}

Submitted on 19 Oct 2015

HAL is a multi-disciplinary open access archive for the deposit and dissemination of scientific research documents, whether they are published or not. The documents may come from teaching and research institutions in France or abroad, or from public or private research centers.
L'archive ouverte pluridisciplinaire HAL, est destinée au dépôt et à la diffusion de documents scientifiques de niveau recherche, publiés ou non, émanant des établissements d'enseignement et de recherche français ou étrangers, des laboratoires publics ou privés. 


\title{
Simulating Patient Specific Multiple Time-point MRIs From a Biophysical Model of Brain Deformation in Alzheimer's Disease
}

\author{
Bishesh Khanal ${ }^{1}$, Marco Lorenzi ${ }^{21}$, Nicholas Ayache ${ }^{1}$ and Xavier Pennec ${ }^{1}$ \\ 1 INRIA Sophia Antipolis Méditerranée, Asclepios Research Project \\ 2004 Route des Lucioles - BP 93, 06902 Sophia Antipolis, France \\ 2 University College London, Translational Imaging Group, \\ London, UK
}

\begin{abstract}
This paper proposes a framework to simulate patient specific structural Magnetic Resonance Images (MRIs) from the available MRI scans of Alzheimer's Disease(AD) subjects. We use a biophysical model of brain deformation due to atrophy that can generate biologically plausible deformation for any given desired volume changes at the voxel level of the brain MRI. Large number of brain regions are segmented in $45 \mathrm{AD}$ patients and the atrophy rates per year are estimated in these regions from the available two extremal time-point scans. Assuming linear progression of atrophy, the volume changes in scans closest to the half way time period is computed. These atrophy maps are prescribed to the baseline images to simulate the middle time-point images by using the biophysical model of brain deformation. From the baseline scans, the volume changes in real middle time-point scans are compared to the ones in simulated middle time-point images. This present framework also allows to introduce desired atrophy patterns at different time-points to simulate non-linear progression of atrophy. This opens a way to use a biophysical model of brain deformation to evaluate methods that study the temporal progression and spatial relationships of atrophy of different regions in the brain with $\mathrm{AD}$.
\end{abstract}

Keywords: Alzheimer's disease, biophysical modeling, biomechanical simulation

\section{Introduction}

Alzheimer's Disease (AD) is one of the most common types of dementia. It is a neurodegenerative disease that progresses gradually over several years with the accumulation of neurofibrillary tangles (NFTs) and amyloid- $\beta$ (A- $\beta$ ) plaques [2]. These microscopic neurobiological changes are followed by the progressive neuronal damage that leads to the atrophy of the brain tissue. The atrophy or the volume changes of brain tissue is a macroscopic change that structural Magnetic Resonance Imaging (MRI) can estimate in different brain regions. Many different methods have been proposed to estimate atrophy in some particular regions of brain that are known to be affected in $\mathrm{AD}[9]$. 
In addition to estimating specific brain structures with atrophy, longitudinal imaging data could also potentially be used to study the temporal interrelationship of atrophy in different structures. For instance in [6], authors estimate per-individual rates of atrophy in 34 cortical regions and in hippocampus. Then they study the groupings of these structures based on the correlation of the atrophy rates. In [8], authors define AD progression as a series of discrete events. Atrophy in different parts of the brain are taken as different events along with clinical events. Without any prior to their ordering, the model finds most probable order for these events from the data itself. They use Bayesian statistical algorithms for fitting in the event-based disease progression model. The objective of these kinds of studies is to understand how different regions of brain interact during the neurodegeneration and find its trajectory. Such studies can benefit with large number of longitudinal images of AD patients. In this context, a model that can simulate many time-point images from a few available longitudinal images can be a valuable tool.

Atrophy simulators [11][14][18][5] have been proposed in the literature and used mostly for the validation of registration or segmentation methods [4][16], or to estimate uncertainty in the measured atrophy [17]. The simulators in [11][14][16] use a Jacobian based methods where the desired level of atrophy is set at each voxel, and the deformation that best approximates the desired level of atrophy is found. Regularization is used in the optimization to enforce certain desired conditions such as topology preservation. The advantage of these methods is the ability to define atrophy maps at the voxel level. However regularization parameters used to enforce topology preservation are generally difficult to relate to a plausible biophysical process of $\mathrm{AD}$ and can create difficulties in simulating opening of certain structures such as sulci. It is not trivial to consider different tissue behaviors in such approaches. In [18][5], authors propose a model of brain deformation based on thermoelasticity. Volume changes are defined in particular structures/tissues of a meshed brain by assigning different thermal coefficients. Simulation of the images is done by first solving the thermoelastic model of tissue deformation with Finite Element Method (FEM), and then by interpolating the obtained displacement field from the mesh to the image. FEM involves moving back and forth from voxels to meshes which creates numerical difficulties and inaccuracies in the model personalization.

In [12] we proposed a new biophysical model of brain deformation due to atrophy in $\mathrm{AD}$ that combines the advantages of the models mentioned in the previous paragraph. The mechanisms of neuronal deaths and its evolution are not well known for $\mathrm{AD}$ and are likely to be primarily guided by complex physiological processes. However we believe that the biomechanics of brain tissue might play an important role in determining the consequence of the neuronal deaths on brain shape changes. Our biophysical model presented in [12] builds upon the assumptions that we relate to the biophysical process of tissue shape changes as the consequence of local volume loss. This model can be used to simulate time-series MRIs starting from a real input baseline MRI. 
In this work we use our biophysical model developed in [12] to present a framework that allows to interpolate or extrapolate patient specific unseen timepoint images from at least two available time-point images of the subject and to assess how closely these simulated trajectories follow real patient trajectories. We also improve the implementation of the boundary condition of the model by imposing zero deformation in the skull and all the regions outside of the skull. In [12] the zero deformation was imposed at the image boundaries and not at the brain-skull boundary.

The following section briefly explains the assumptions and implementation of the biophysical model we presented in [12], and in section 3 we present how we interpolate new images between two acquisition time points.

\section{Biophysical Model of Brain Deformation due to Atrophy}

The atrophy rate $\tilde{a}(\boldsymbol{x}, t)$ at any position $\boldsymbol{x}$ at time $t$ for a representative elementary volume of $V(\boldsymbol{x}, t)$ is defined as the negative rate of change of volume per unit volume:

$$
\tilde{a}=\frac{-1}{V} \frac{\partial V}{\partial t}
$$

For any time $\Delta t$ that results in sufficiently small displacement, the amount of atrophy is $a=\tilde{a} \Delta t$. Any deformation field that has atrophy $a$ should satisfy the following equation:

$$
\nabla \cdot \boldsymbol{u}=-a,
$$

where $u$ is the displacement of material particles during the $\Delta t$.

We do not explicitly model the neuronal loss and tissue remodeling at the microscopic level which requires biochemical and cellular physiological knowledge in detail. We abstract the phenomenon that evolves over several months or years in the brain. In Creutzfeldt-Jakob disease, no gross brain shape changes are reported and the imaging only shows hyperintense signals on T2-weighted images [10]. However, this is not the case in AD and longitudinal MRIs show a decrease of brain volume instead [9] without any "holes". That means the tissues should restructure as the neuronal deaths increase with time. This leads us to a basic assumption in the proposed model that after the death of neurons, remodeling of the tissue occurs such that the tissue density remains constant while both the mass and volume decrease. We further assume that the atrophy creates internal stress which results in the deformation minimizing a strain energy.

Using Saint Venant-Kirchoff model for an elastic material, this can be expressed as the minimization of which results in the deformation of the tissue minimizing the strain energy.

$$
R(\boldsymbol{u}, p)=\int \mu \operatorname{tr}\left(\boldsymbol{E}(\boldsymbol{u})^{2}\right)+\frac{\lambda}{2}(\operatorname{tr}(\boldsymbol{E}(\boldsymbol{u})))^{2}-\int p(\nabla \cdot \boldsymbol{u}+a)
$$


where $p$ is a Lagrange multiplier, $\mu$ and $\lambda$ are Lamé constants, and $\boldsymbol{E}$ is Langrangian Green strain defined as: $\boldsymbol{E}=\frac{1}{2}\left(\nabla \boldsymbol{u}+\nabla \boldsymbol{u}^{T}+\nabla \boldsymbol{u}^{T} \nabla \boldsymbol{u}\right)$.

By taking a sufficiently small time step $\Delta t$, this deformation could be reasonably modeled as being linear elastic. For example, for a $2 \%$ global atrophy per year, we have $\Delta t=1$ year, and the atrophy during the year as $a=0.02$.

Under linear elastic assumptions, minimizing the energy in equation (2) is equivalent to solving the following set of equations.

$$
\begin{aligned}
\mu \Delta \boldsymbol{u}-\nabla p & =(\mu+\lambda) \nabla a \\
\nabla \cdot \boldsymbol{u} & =-a
\end{aligned}
$$

where $\Delta \boldsymbol{u}$ is a component-wise Laplacian of $\boldsymbol{u}$. This equation is very similar to the Stokes flow equation in fluid dynamics. The difference is in the non-zero divergence term which corresponds the loss of mass and volume in the tissue. The momentum equation shows that the gradient of the prescribed volume loss acts as the force term that moves the tissue for the structural remodeling. The Lagrange multiplier $p$ is some sort of virtual pressure whose algebraic values can be seen as the sources and sinks of fluid.

\subsection{Modeling CSF Region}

The timescale of CSF production is hours, which is much smaller compared to the time scale of tissue remodeling due to atrophy. To allow the CSF to expand as required when the brain deforms due to the prescribed atrophy, we release the strict incompressibility constraint in 3 . Furthermore, the force term of the momentum equation in 3 is no longer required. Thus the combined equation for both the brain parenchyma and the CSF regions is:

$$
\begin{aligned}
\mu \Delta \boldsymbol{u}-\nabla p & =(\mu+\lambda) \nabla f \\
\nabla \cdot \boldsymbol{u}+k p & =-f
\end{aligned}
$$

where we have,

- Brain parenchyma region: $k=0$, and $f=a$

- CSF region: $k=1$, and $f=0$.

Boundary Conditions: Dirichlet boundary conditions with zero displacement is enforced at the skull.

Material Parameters $\mu$ and $\lambda$ : The deformation model here corresponds to the structural readjustments due to cell loss, thus the Lamé parameters do not have the same usual meaning as during an elastic deformation of the material due to application of an external load/force. The voxel-wise volume change constraint and the boundary conditions i.e. the shape of the tissue-CSF and brain-skull interface have much more impact on the deformation of the brain parenchyma than any specific scalar values of $\mu$ and $\lambda$. In the present work these coefficients are set to 1 and 0 respectively. 


\subsection{Staggered Grid Discretization and Finite Difference Method}

The equation (4) requires a partition of the computational domain into different regions. These regions are obtained by using skull stripping and segmentation of the input baseline brain MRI. The solution of the PDE provides us a deformation field that is applied to the baseline image to generate simulated follow-up image. We use Finite Difference Method (FDM) with staggered grid discretization to solve the system of PDEs in (4). Using staggered grid with proper placing of the pressure and velocity variable ensures stability in the solution. FDM is chosen instead of FEM to avoid brain meshing and the complexity of transporting computed variables from mesh to image at each iteration. This allows us to solve the system in a grid that is of the same size as the input image where the grid fits naturally to the image. This also makes it easier to obtain the partition of the computational domain into different regions directly by using a skull stripping and a segmentation algorithm.

For typical brain MRIs of $1 \mathrm{~mm}^{3}$ resolution, this computational problem size becomes so large that direct solvers are impractical due to memory limitations. The set of equations in 4 is similar to Stokes flow equation which is a saddle point system. It needs a suitable combination of an iterative solver and a preconditioner to solve it. We use a Schur factorization to split the equations into the momentum equation and the pressure equation. Each of these equations is solved using different iterative solvers. Our implementation uses composable solvers for multiphysics with PETSc library [1] using fieldsplit preconditioner, an approach detailed in [3] with an example for Stokes flow solver with Schur complement factorization. The momentum equation is preconditioned with hypre which is an algebraic multigrid preconditioner and can be called from the PETSc interface. The implemented system is run using distributed computing in a locally available cluster.

\section{Experiments and Results}

We use the Miriad dataset [13] that has multiple time-point T1 structural MRIs of 45 Alzheimer's patients in the range of 2 weeks to 2 years. Since the dataset contains several time-point scans, we can compare the simulated intermediate time-point images to the corresponding real intermediate images. To prescribe personalized atrophy patterns we need an atrophy estimation for each subject from the extremal time-points. We perform the whole brain segmentation using recon-all command in FreeSurfer [7]. For the segmentation, FreeSurfer's longitudinal stream [15] is used to create unbiased subject specific templates. This allows us to compare the volumes of large number of regions in the baseline and the follow-up images and estimate atrophy in each of these regions. These estimated atrophy can then be modified and prescribed to each of the baseline MRIs to predict intermediate time-point images. The setup of the experiment we performed is described as follows: 


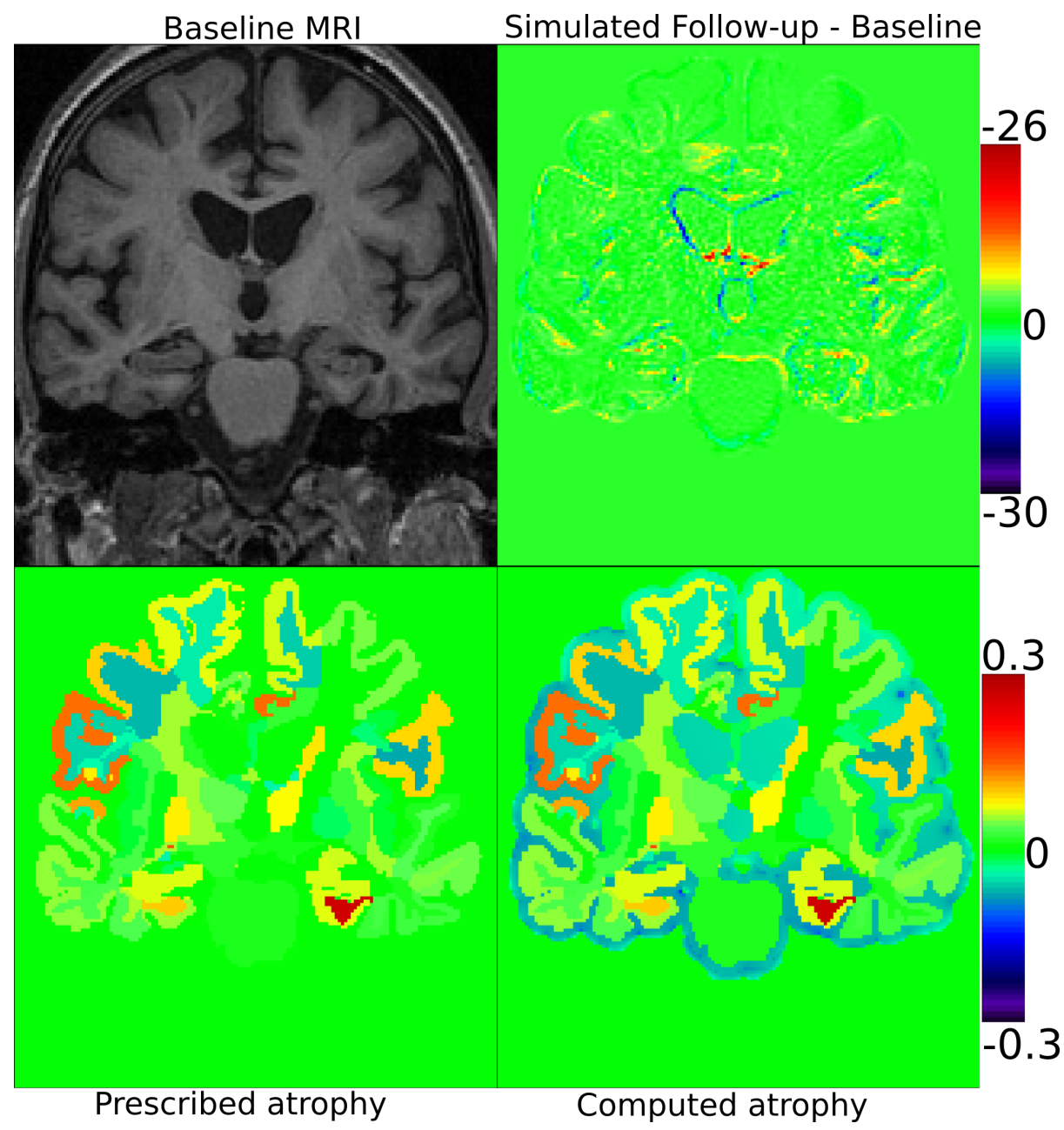

Fig. 1. Top left is the input baseline MRI to which the atrophy shown in bottom left is prescribed. A follow-up image is simulated using our model. The difference of the simulated follow-up and the baseline MRI is shown in top right. Bottom right is the atrophy map associated to the deformation field that was obtained as the solution of the model when using the atrophy map on the left as input. As expected, in brain tissue region they are same while in CSF there is expansion to compensate the tissue loss keeping the skull fixed.

1. Find available extremal time-point scans: baseline $I_{b}$ and the final follow-up $I_{f}$. Let $t_{f}$ be the time (in years) between the baseline scan and the final scan.

2. Find a mid-point scan $I_{m}$ that was scanned $t_{m}$ years after the first scan. This is found by finding $t_{m}$ that is closest to $t_{f} / 2$. 
3. Use FreeSurfer to estimate an atrophy map $a_{f}$. This is a scalar image such as the one shown in bottom left of Figure 1. The intensities are the atrophy estimated from FreeSurfer for all the segmented brain regions.

4. Simulate a follow-up image $\hat{I}_{m}$ that corresponds to the mid-point scan $I_{m}$ by prescribing $a_{m}$ where $a_{m}=a_{f} * t_{m} / t_{f}$.

5. Run the FreeSurfer whole brain segmentation on this simulated image $\hat{I}_{m}$ and compute volumes of all the segmented regions.

6. Compare FreeSurfer computed volumes of all regions of the images $I_{m}$ and $\hat{I}_{m}$.

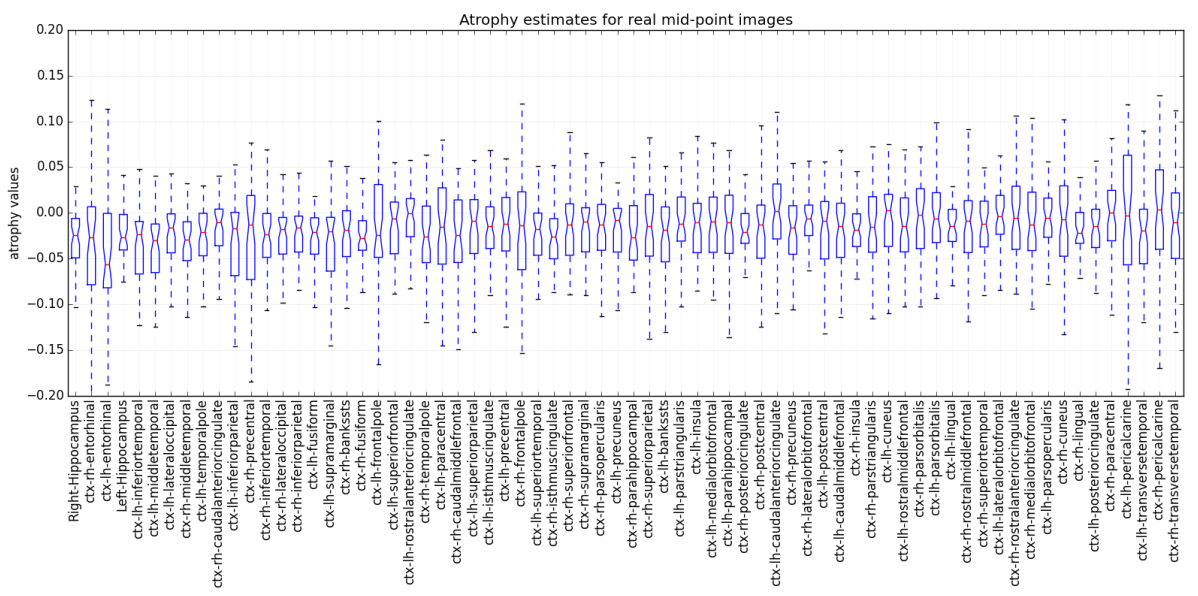

Fig. 2. Boxplot of the atrophy estimates for the real mid-point images in the coritcal regions and hippocampus. These are the regions that were used in [6]. The data shows the distribution of FreeSurfer atrophy estimates in the AD population of the MIRIAD dataset when considering the first and the mid-point scans.

In Figure 3 we see that for most regions the difference in the atrophy estimation of the interpolated mid-point image and that of actual mid-point image have median close to zero. Higher variability in the difference seems to be mostly in the regions where there is higher variability in the atrophy estimates of the real mid-point images. The large inter-subject variation of the difference between the atrophy estimate in the real mid-point image and the interpolated mid-point image could be due to several reasons. One obvious issue is that the FreeSurfer segmentation with the longitudinal stream expects all the images that are to be segmented to be preprocessed in the same manner. However, in our case the interpolated mid-point image has undergone an extra resampling step while the real mid-point image has not. This extra resampling step is required because the interpolated mid-point image was obtained by warping the real baseline image with a displacement field. Furthermore, the choice of interpolation during 


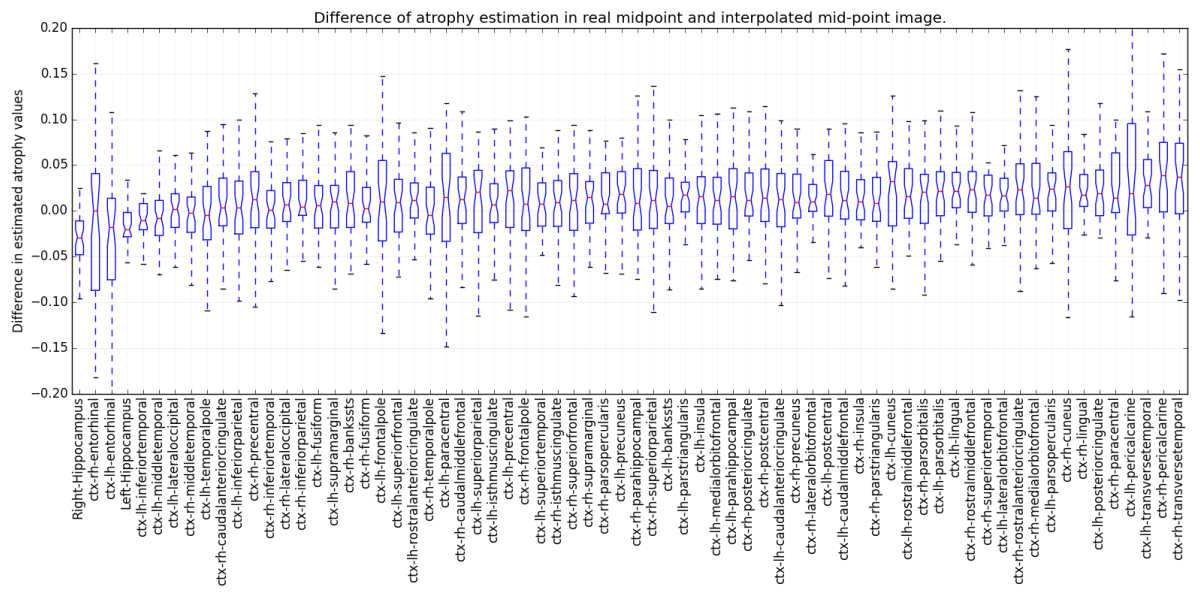

Fig. 3. Boxplot of the difference in the FreeSurfer atrophy estimate in the real midpoint image and the interpolated mid-point image for all the $45 \mathrm{AD}$ subjects present in MIRIAD dataset. The regions shown are the same as the one shown in Figure 2 and are displayed in the same order. The interpolated mid-point image is obtained by simulation using the pair of extremal time-point images of each subject.

the resampling step can also affect the volume measurements by FreeSurfer. We used trilinear interpolation for the resampling. The extra resampling step and the choice of interpolation does have an effect on the estimation of volumes. This has been shown, for instance in [16] for other segmentation based atrophy estimation techniques. Furthermore, the interpolated mid-point image has a noise (noise inherent in any MRI) that is highly correlated with the real baseline image. However, the noise in real mid-point image is not correlated to the baseline image. This also affects the atrophy estimation and hence contributes to the variability in the atrophy estimation difference. A detailed analysis must be done to find out the regions that are the most reliable ones in estimating volume changes for both the real and simulated images. The performance of the atrophy measurement tools on simulated images should be thoroughly evaluated to find out the best regions that we can rely upon to test how closely we predict volume changes in new time-point images.

In this case we have interpolated the intermediate time-point by linearly scaling the estimated atrophy. For a small time window of a couple of years this is reasonable but if we want to extrapolate for instance for several years we would need a non-linear model of atrophy progression. The presented framework allows one to compare the trajectory of brain shape changes with different models of atrophy progression. The ability to prescribe any desired atrophy at any time point allows one to introduce atrophy at different regions of brain at different times. This can be exploited in evaluating the methods proposed in studies such as [8] which order the events from time-series data. 


\section{Conclusions}

We have proposed a framework to generate patient specific multiple time-point images based on our biophysical model of brain deformation due to atrophy in AD. The used model is motivated from biomechanical principles and it models the consequence of tissue loss in brain shape changes. From the available two scans of MRI of a patient at two different time-points we estimate atrophy in large number of brain structures using FreeSurfer whole brain segmentation [7]. The derived atrophy patterns are linearly scaled and prescribed to the biophysical model to simulate the another time-point image. Using the MIRIAD dataset [13] of $45 \mathrm{AD}$ subjects with multiple time-points we compare the simulated time-point images against the actual time-point images. The future works will include building the most reliable methods to compare the volumes in simulated and real images. We will also explore the possibility of evaluating methods that study the temporal relationships, ordering and co-evolution of atrophy in different structures of the brain.

Acknowledgements Part of this work was funded by the European Research Council through the ERC Advanced Grant MedYMA 2011-291080.

\section{References}

1. S. Balay, J. Brown, K. Buschelman, W. D. Gropp, D. Kaushik, M. G. Knepley, L. Curfman McInnes, B. F. Smith, and H. Zhang. PETSc Web page, 2013. http://www.mcs.anl.gov/petsc.

2. H. Braak and E. Braak. Neuropathological stageing of Alzheimer-related changes. Acta Neuropathologica, 82(4):239-259, sep 1991.

3. J. Brown, M.G. Knepley, D.A. May, L.C. McInnes, and B. Smith. Composable Linear Solvers for Multiphysics. In 2012 11th International Symposium on Parallel and Distributed Computing (ISPDC), pages 55-62, jun 2012.

4. O. Camara, R. I. Scahill, J. A. Schnabel, W. R. Crum, G. R Ridgway, D. LG Hill, and N. C. Fox. Accuracy Assessment of Global and Local Atrophy Measurement Techniques with Realistic Simulated Longitudinal Data. In N. Ayache, S. Ourselin, and A. Maeder, editors, MICCAI 200\%, volume 4792 of LNCS, pages 785-792. Springer, Heidelberg, 2007.

5. O. Camara, M. Schweiger, R. I. Scahill, W. R. Crum, B. I. Sneller, J. A. Schnabel, G. R. Ridgway, D. M. Cash, D. L. G. Hill, and N. C. Fox. Phenomenological model of diffuse global and regional atrophy using finite-element methods. IEEE Transactions on Medical Imaging, 25(11):1417-30, nov 2006.

6. Owen Carmichael, Donald G. McLaren, Douglas Tommet, Dan Mungas, and Richard N. Jones. Coevolution of brain structures in amnestic mild cognitive impairment. NeuroImage, 66:449-456, feb 2013.

7. Bruce Fischl, David H. Salat, Evelina Busa, Marilyn Albert, Megan Dieterich, Christian Haselgrove, Andre van der Kouwe, Ron Killiany, David Kennedy, Shuna Klaveness, Albert Montillo, Nikos Makris, Bruce Rosen, and Anders M. Dale. Whole Brain Segmentation: Automated Labeling of Neuroanatomical Structures in the Human Brain. Neuron, 33(3):341-355, jan 2002. 
8. Hubert M. Fonteijn, Marc Modat, Matthew J. Clarkson, Josephine Barnes, Manja Lehmann, Nicola Z. Hobbs, Rachael I. Scahill, Sarah J. Tabrizi, Sebastien Ourselin, Nick C. Fox, and Daniel C. Alexander. An event-based model for disease progression and its application in familial Alzheimer's disease and Huntington's disease. NeuroImage, 60(3):1880-1889, apr 2012.

9. G. B. Frisoni, N. C. Fox, C. R. Jack, P. Scheltens, and P. M. Thompson. The clinical use of structural MRI in Alzheimer disease. Nature Reviews. Neurology, 6(2):67-77, feb 2010.

10. R. T. Johnson and C. J. Gibbs Jr. Creutzfeldt-Jakob disease and related transmissible spongiform encephalopathies. New England Journal of Medicine, 339(27):1994-2004, 1998.

11. B. Karaçali and C. Davatzikos. Simulation of tissue atrophy using a topology preserving transformation model. IEEE Transactions on Medical Imaging, 25(5):64952, may 2006.

12. Bishesh Khanal, Marco Lorenzi, Nicholas Ayache, and Xavier Pennec. A Biophysical Model of Shape Changes due to Atrophy in the Brain with Alzheimer's Disease. In Polina Golland, Nobuhiko Hata, Christian Barillot, Joachim Hornegger, and Robert Howe, editors, Medical Image Computing and Computer-Assisted Intervention - MICCAI 2014, volume 8674 of Lecture Notes in Computer Science, pages 41-48. Springer International Publishing, 2014.

13. Ian B. Malone, David Cash, Gerard R. Ridgway, David G. MacManus, Sebastien Ourselin, Nick C. Fox, and Jonathan M. Schott. MIRIAD-Public release of a multiple time point Alzheimer's MR imaging dataset. NeuroImage, 70:33-36, apr 2013.

14. P. Pieperhoff, M. Südmeyer, L. Hömke, K. Zilles, A. Schnitzler, and K. Amunts. Detection of structural changes of the human brain in longitudinally acquired MR images by deformation field morphometry: methodological analysis, validation and application. NeuroImage, 43(2):269-87, nov 2008.

15. Martin Reuter, Nicholas J. Schmansky, H. Diana Rosas, and Bruce Fischl. Withinsubject template estimation for unbiased longitudinal image analysis. NeuroImage, 61(4):1402-1418, jul 2012.

16. S. Sharma, V. Noblet, F. Rousseau, F. Heitz, L. Rumbach, and J.P. Armspach. Evaluation of brain atrophy estimation algorithms using simulated ground-truth data. Medical Image Analysis, 14(3):373-89, jun 2010.

17. S. Sharma, F. Rousseau, F. Heitz, L. Rumbach, and J.P. Armspach. On the estimation and correction of bias in local atrophy estimations using example atrophy simulations. Computerized Medical Imaging and Graphics, 37(7-8):538 - 551, 2013.

18. A. D. C. Smith, W. R. Crum, D. L. Hill, N. A. Thacker, and P. A. Bromiley. Biomechanical simulation of atrophy in MR images. In Medical Imaging 2003, pages 481-490. International Society for Optics and Photonics, 2003. 